Kapoor et al. Oral Health Correlates of Captivity

\title{
1 Oral Health Correlates of Captivity
}

3 Varsha Kapoor ${ }^{\mathrm{a}}$, Tyler Antonelli ${ }^{\mathrm{b}}$, Jennifer A. Parkinson ${ }^{\mathrm{c}}$, Adam Hartstone-Rose ${ }^{\text {bd* }}$

$4 \quad{ }^{a}$ Medical University of South Carolina School of Dental Medicine

$5 \quad{ }^{\mathrm{b}}$ Department of Cell Biology and Anatomy, University of South Carolina School of Medicine

$6 \quad{ }^{c}$ Department of Anthropology, University of San Diego

$7 \quad$ d Animal Collections and Conservation, Riverbanks Zoo and Garden

8 Running Head: Oral Health Correlates of Captivity

9 *Corresponding author: Ph: 803-216-3816, F: 803-216-3846, AdamHR@ @s.edu 


\section{ABSTRACT}

The predominant diet fed to captive carnivores in North America consists of ground meat

13 formulated to provide full nutritional requirements. However, this ground meat diet completely

14 lacks the mechanical properties (i.e., toughness and hardness) of the foods these animals would

15 consume in the wild. The goal of this study is to evaluate the effect of captivity on oral health by

16 comparing the prevalence of periodontal disease and dental calculus accumulation in wild and

17 captive lions and tigers (Panthera leo and P. tigris), and to also correlate oral health with cranial

18 morphology in these specimens.. To achieve this, 34 adult lion and 29 adult tiger skulls were

19 scored for the presence and extent of dental calculus and periodontal disease. These oral health

20 scores were also compared to cranial deformations examined in a previous study. We found that

21 the occurrence and severity of calculus buildup and periodontal disease was significantly higher

22 in captive felids compared to their wild counterparts. Further, higher calculus accumulation

23 occurred on the posterior teeth when compared to the anterior teeth, while an opposite trend for

24 periodontal disease was observed. We also found a significant correlation between oral health and

25 cranial morphology of lions and tigers. The results suggest that food mechanical properties are

26 significant factors contributing to oral health in felids.

28 KEY WORDS: Panthera, P. leo, P. tigris, gingivitis, gum disease, caries

\section{INTRODUCTION}

30 The composition of the diet of captive carnivores is of great interest to animal curators,

31 keepers and veterinarians. However, this interest has focused almost entirely on the nutritional

32 composition of the diet (e.g., Whitehouse-Tedd et al., 2015) and little consideration has been

33 given to the mechanical properties of foods such as toughness and hardness. While the

34 incorporation of bones as dietary supplements or as enrichment is a growing trend (McPhee,

35 2002; Skibiel et al., 2007), the predominant diet fed to captive carnivores in North America 
Kapoor et al.

consists of ground meat (Bechert et al., 2002; Kerr et al., 2013). While ground meat diets may

37 contain all of the nutrients found in a natural diet, they does not simulate the ingestive or

38 masticatory challenges that wild carnivores face (Haberstroh et al., 1984). In particular, the lack

39 of bone and connective tissue in the captive diet may lead to dental health issues (Haberstroh et

40 al., 1984; Lindburg, 1988). Numerous studies in domestic animals have demonstrated that

41 mechanical attributes of the diet appear to affect dental calculus buildup and oral health in general

42 (Antonelli et al., 2015; Logan, 2006; Vosburgh et al., 1982; Watson, 1994) however, thorough

43 examination of the way in which the mechanical properties of foods affect oral health in large

44 felids remains to be carried out. This study focuses on the correlation between dental health and

45 captivity status in captive felids.

\section{BACKGROUND}

\section{$47 \quad$ Experimental Studies}

48 The mechanical properties of food have long been thought to affect the oral health of

49 domestic dogs and cats as well as captive exotic carnivores (Fagan, 1980). Several studies have

50 noted the correlation of soft diets with excessive calculus accumulation, gingivitis, and

51 periodontal disease in dogs and cats (Logan, 2006; Watson, 1994) and exotic carnivores (Fagan,

52 1980). It is thought that a dry diet is associated with less gingival pathology because the abrasive

53 action of dry/rough foods against the gingivae in effect acts as a toothbrush, cleaning the teeth

54 and removing food particles trapped in the gingival sulcus (Haberstroh et al., 1984; Vosburgh et

55 al., 1982)

56 Numerous studies have supported the hypothesis that mechanical properties of the diet

57 are significant factors in the oral health of captive carnivores. A study conducted on captive

58 wolves (Canis lupus) found that animals fed a hard, dry diet exhibited significantly lower levels

59 of plaque accumulation after four months than those fed a soft, meat-based diet (Vosburgh et al.,

60 1982). A study of captive Amur Tigers (Panthera tigris altaica) found that when animals being 
61 fed a diet of commercially produced soft meat had their diets supplemented at least twice per

62 week with beef bones (with muscle and connective tissue still attached) they showed lower

63 incidence of gingivitis and periodontal disease (Haberstroh et al., 1984). Several studies of

64 domestic beagles (C. lupus familiaris) have also found that those fed abrasive diets including

65 commercial dry foods or biscuits showed lower rates of periodontal disease and tartar

66 accumulation than those on soft diets (Brown and Park, 1968; Samuelson and Cutter, 1991;

67 Watson, 1994). Another study showed that ferrets (Mustela putorius furo) fed a soft diet of bread

68 and milk developed periodontal disease within 8-12 weeks. The addition of bones to their diet

69 prevented periodontal disease and even caused a decrease in calculus accumulation (Verstraete,

70 2003).

\section{Felid Dental Anatomy and Function}

As in all mammals, carnivorans have four types of teeth: incisors, canines, premolars and

74 molars. In most carnivores the incisors are used for grasping and tearing, though they are

75 relatively vestigial in modern felids. Felid canines are used for capturing and killing prey, and the

76 premolars and carnassials are used primarily for slicing flesh. Felids have no functional dental

77 region distal to their carnassials - a molar region that many other carnivorans use for grinding

78 tougher foods (Hartstone-Rose, 2008; Hartstone-Rose et al., 2012; Logan, 2006; Van

79 Valkenburgh, 1989).

80 A typical adult felid has a total of 30 teeth with the following dental formula: I3/3, C1/1,

$81 \mathrm{P} 3 / 2, \mathrm{M} 1 / 1$, where I, C, P and M represent Incisors, Canines, Premolars and Molars respectively,

82 and the number on either side of the " " signifies the number of teeth of that kind in each upper

83 and lower quadrant respectively (Wiggs and Bloom, 2003). Teeth are anchored into the alveolar

84 bone by the periodontal ligament. In healthy animals, gingival tissue covers the alveolar bone and

85 tooth root to a level just below the area where enamel meets the root surface (Niemiec, 2008a).

86 


\section{Dental Calculus and Periodontal Disease}

Dental calculus, or tartar, can be defined as calcified or calcifying deposits of dental

89 plaque (a microbial biofilm) attached to the enamel surface (Busscher et al., 2004). Calculus is

90 primarily composed of inorganic compounds consisting of crystalline salts, but it is usually

91 covered by an active layer of bacterial plaque. New calculus is formed as this active layer of

92 bacterial plaque mineralizes (Deinzer et al., 2005; Marcenes and Sheiham, 1992). When plaque

93 and calculus spread under the gingival line, it causes irritation leading to an inflammatory

94 condition of the soft tissue known as gingivitis (Campbell, 2006). As calculus builds under the

95 gingiva, the bacteria in the sub-gingival plaque set in motion a cycle of damage to the supporting

96 tissues around the tooth, and a more severe form of periodontal disease known as periodontitis

97 may develop (Pihlstrom et al., 2005). Periodontitis can lead to bone loss, tissue destruction, and

98 ultimately tooth loss (Campbell, 2006).

99 Periodontal disease is the most prevalent disease in domestic carnivores and has been

100 found in approximately $80 \%$ of adult dogs in one study (Niemiec, 2008b). It is less common in

101 wild carnivores (Wiggs and Bloom, 2003), possibly due to differences in the food consistency of

102 wild and captive carnivores.

\section{Captive Felid Diet}

105 The most common diet provided to felids in captivity in North America consists almost

106 entirely of commercially prepared meat supplemented with vitamins (Vester et al., 2008). While

107 this ground meat diet attempts to ensures that an animals' nutritional needs are met (Kerr et al.,

108 2013), it lacks the mechanical properties of the natural felid diet which consists of intact prey

109 animal carcasses. In the wild, large felids consume flesh straight from the bone. In doing so, they

110 also consume tough connective tissue and create contact between the surfaces of their teeth and

111 bones from the carcass (Schaller, 1972; Sunquist and Sunquist, 2002). The presence of these

112 natural dietary components is often ignored, if not discouraged in captive feeding. Milliken Meat 
113 Products Ltd., one of the largest producers of carnivore meat diets (under the brand name

114 “Toronto Zoo Carnivore Diets"), advertises that their meat product contains "no bones, cartilage,

115 organs, skin or connective tissues" (Roberts and Gittleman, 1984). The primary reason for this

116 aversion to other carcass parts is likely that these components present a choking hazard to the

117 animals. Secondly, feeding captive carnivores fully intact carcasses can incite public relations

118 problems, as was recently seen at the Copenhagen Zoo where one of the zoo's giraffes was

119 euthanized and fed to the zoo's lions (Bowers, 2014; Schwarts, 2014). Commercially available

120 ground meat diets with added vitamins meet the basic nutritional requirements of captive felids

121 (Vester et al., 2008), but the lack of complete macro-structural elements leaves the captive diet

122 devoid of the mechanical challenges found in the diets of wild carnivores.

\section{Specific Aims and Hypotheses}

125 The overall goal of this study is to assess the effect of captivity on the oral health of

126 felids. Previous research has shown that captivity can negatively affect oral health in other

127 carnivorans (Antonelli et al., 2015), likely due to a soft diet, and given that captive felids in North

128 America are predominantly fed ground meat, we hypothesize that they will have higher

129 incidences of dental calculus and periodontal disease compared to wild felids. Since tooth

130 function differs across regions of the mouth, we also hypothesize that the prevalence of calculus

131 and periodontal disease will also differ between the anterior and posterior teeth. Lastly, we

132 hypothesize that oral health measures will correlate positively with an index of skull deformity

133 that has been found to be a marker of captivity (Hartstone-Rose et al., 2014).

\section{MATERIALS and METHODS}

\section{Sample}

136 We collected data on 63 adult lion (Panthera leo) and tiger (P. tigris) skulls (Table 1)

137 from the American Museum of Natural History (AMNH; New York), and the Smithsonian 
Institution's National Museum of Natural History $\left(\mathrm{NMNH}^{1}\right.$ Washington DC) as well as several

139 specimens in the comparative anatomy research collection at the University of South Carolina

140 School of Medicine. Specimens were identified as adults by the presence of fully erupted

141 permanent teeth. The wild specimens were collected throughout the natural range of each species

142 and the captive animals were accessioned from The Barnum and Bailey Circus (traveling

143 throughout the USA), the Carolina Tiger Rescue (Pittsboro, NC), National Zoological Park

144 (Smithsonian, Washington DC), Toledo Zoological Society (Toledo, OH), and several historical

145 zoos that now fall under the Wildlife Conservation Society (all around New York City): the

146 Bronx Zoo, Central Park Zoo, New York Park Commission, New York Zoo, New York Zoo

147 Gardens, New York Zoo Society, and the Prospect Park Zoo.

148 The sample population of specimens was sorted according to species and captivity status.

149 For the sake of this paper, "wild" refers to individuals with known wild geographic origin and

150 "captive" refers to individuals who resided in zoos or animal rescue facilities at the time of their

151 deaths. Although captivity status at birth is unknown for most captive specimens in our sample,

152 because importation of wild animals has historically been rare, it can safely be presumed that

153 most if not all were born in captivity.

155 Insert Table 1 Here

All specimens were scored for calculus and periodontal disease by assessing the

158 dental arcade in six sections (Fig. 1): upper and lower cheek teeth (premolars and molars)

159 on the left and right side separately and upper and lower anterior teeth (canines and

160 incisors). Each section was scored from 0 to 5 for calculus buildup and periodontal

161 disease (see tables 2 and 3 for explanation of indices). Each score represents the average

162 score for all teeth in each of the six sections. Using the average score for a section rather

${ }^{1}$ Specimens from the NMNH are cataloged with the designation "USNM". 
Kapoor et al. Oral Health Correlates of Captivity

163 than scoring each individual tooth eliminated the discrepancies that might arise, for

164 example, due to individual missing or damaged teeth.

165

166 Insert Figure 1 Here

167

168 Calculus Scoring

169 The amount of calculus covering the crowns of all teeth in each of the six sections (Fig.

170 1) was observed and scored according to the calculus index outlined in Table 2 . This index was

171 created specifically for the goals of our analysis, but it is based on previously developed similar

172 scales for scoring human dentition (Deinzer et al., 2005; Dog, 2005; Muhvic-Urek et al., 2007).

173

174 Insert Table 2 Here

175

176 Periodontal Disease Scoring

177 Specimens were scored for periodontal disease by assessing the presence and

178 degree of bone porosity relative to the distance from the alveolar margin using a scale

179 developed for the purposes of this analysis (Table 3). As in the scoring for calculus build

180 up, the average score for all teeth in each section was taken.

181

182 Insert Table 3 Here

183

184 Cranial Deformation Score

185 Hartstone-Rose and colleagues (2014) used three-dimensional geometric morphometric 186 analyses of the same specimens that we have used in the present study to assess the correlation

187 between skull shape distortions and captivity status. In their principal component analysis of 43

188 cranial landmarks, the second greatest source of morphological variation across the population - 
Kapoor et al. $\quad$ Oral Health Correlates of Captivity

189 second only to the variation separating the two species - sorted the sample according to captivity

190 status. This factor (the second principal component) accounted for $14.81 \%$ of the morphological

191 variation in skull shape - almost twice as much of the variation as the axis that sorted these

192 specimens by sex (which contributed $7.97 \%$ of the variation). Through this analysis and analyses

193 of other specific measures, Hartstone-Rose and colleagues found that the degree of skull

194 deformity is highly correlated with captivity status, and thus in the present study we use this

195 second principal component score as a "captivity coefficient". We compare this captivity

196 coefficient to our periodontal disease and calculus scores to evaluate the correlation between the

197 captivity-driven cranial deformation and oral health.

199 Statistical Analyses

200 Statistical analyses were conducted using JMP statistical software (version 10.0.2; SAS).

201 Mean and maximum values for periodontal disease and calculus scores were used to examine

202 correlations between oral health scores and captivity status, taxon, captivity coefficient, and

203 variation between different regions of the mouth. One-way ANOVA tests were conducted to

204 determine if captive animals have significantly higher periodontal and calculus scores than wild

205 animals.

206

207 RESULTS

208 Effect of Captivity Status on Oral Health

209 The occurrence and magnitude of periodontal and calculus scores were significantly

210 higher in captive animals compared to their wild counterparts.

211 To examine simply whether there was an increased presence or absence of

212 substantial periodontal disease and calculus accumulation in captive animals, we

213 calculated the total percentage of wild and captive specimens that have some or 
Kapoor et al. Oral Health Correlates of Captivity

214 moderate-to-extreme (score of $>0$ or $>1$ respectively) calculus and periodontal disease

215 scores. While almost none of the wild felids exhibited moderate or severe periodontal

216 disease and calculus, the opposite was true of the captive felids (Table 4 and figure 2).

218 Insert Table 4 Here

219 Insert Figure 2 Here

To examine not only the presence or absence of calculus and periodontal disease, but also

222 the magnitude of these dental health variables, we conducted a one-way ANOVA test (Table 5).

223 Results show that the mean and maximum periodontal score and mean and maximum calculus

224 score statistically separate captive and wild populations, with captive animals showing

225 significantly higher $(\mathrm{p}=<.0001)$ scores for both periodontal disease and calculus accumulation.

226 Furthermore, the captive populations have wider standard deviations across these variables,

227 demonstrating higher variability in these measures of oral health in captive felids.

\section{Insert Table 5 Here}

231 Calculus and periodontal disease were also analyzed exclusively in the two anterior sections

232 (upper and lower). Results from these one-way ANOVA tests were significant for both calculus

233 and periodontal disease in both upper and lower anterior teeth (Table 6), with the captive

234 specimens scoring higher in every analysis. The results were particularly pronounced in regards

235 to periodontal disease where the captive specimens scored an average of 2.60 and 2.11 for upper

236 and lower anterior, respectively. Conversely, the wild specimens scored averages of 0.36 for

237 upper and 0.71 for lower anterior teeth. 
Kapoor et al. $\quad$ Oral Health Correlates of Captivity

In order to examine the correlation between oral health and overall health, we compared

241 the captivity coefficient scores from Hartstone-Rose and colleagues (2014) to mean/maximum

242 periodontal disease, and mean/maximum calculus scores. Since neither the captivity coefficient

243 nor the oral health scores are obviously "independent" variables (i.e., should be regarded with

244 assumptions of error), to compare them, we used Reduced Major Axis regression (RMA) rather

245 than Ordinary Least Square (OLS). Results showed a statistically significant relationship between

246 oral health and cranial morphology (Table 7). Bivariate fits of mean and maximum periodontal

247 score and mean and maximum dental calculus score against captivity coefficient showed a

248 positive correlation. Higher captivity coefficient scores are correlated with higher periodontal

249 disease and dental calculus scores. The relationship between the mean and maximum periodontal

250 score and mean and maximum calculus score versus captivity coefficient is also statistically

251 significant.

252

253 Insert Table 7 Here

254

255 Differences between Posterior vs. Anterior Dentition and Oral Health

256 There is a significant relationship between the oral health scores of anterior and posterior

257 teeth (Table 8). However, the slopes of the anterior and posterior calculus and periodontal disease

258 scores are not isometric; the periodontal disease score regression is negatively allometric and the

259 calculus score regression is positively allometric. Thus, there is significantly more periodontal

260 disease found in the anterior dentition, but significantly more calculus found in the posterior

261 dentition.

262

263 Insert Table 8 Here

265 Influence of Sex and Species on Oral Health 
In order to assess the influence of sex and species on periodontal and calculus scores, one-way ANOVA tests were conducted comparing species (tiger versus lion), and

268 sex (male versus female) with mean and maximum periodontal disease and calculus

269 scores. Only the comparison of maximum periodontal disease by species was statistically

270 significant $(\mathrm{p}=0.038)$ with tigers having the greater score. To negate a sampling bias

271 wherein the ratio of captive specimens to wild specimens was much higher in tigers

272 (20:9) than in lions (15:19) and slightly in males (19:12) than in females (16:16), sex and

273 species were divided by captivity status (wild tiger versus wild lion, captive tiger versus

274 captive lion, wild males versus wild females, and captive males versus captive females)

275 and then subjected to one-way ANOVA tests. Results from these tests found no

276 significant differences in either species or sex.

\section{DISCUSSION}

\section{Effect of Captivity on Oral Health}

279 The goal of this study was to evaluate the occurrence of periodontal disease and the degree

280 of calculus accumulation in wild and captive felids. Our results indicate that for felids held in

281 North America, captivity has a strong effect on the oral health of lions and tigers. We predicted

282 the incidence and magnitude of dental calculus and periodontal disease would be higher in 283 captive felids, which are traditionally fed a soft diet in North America. As expected, a higher 284 incidence of both was observed in the captive animals compared to their wild counterparts, and 285 the magnitude of those markers of poor oral health was also significantly higher in captive 286 animals.

287 Since the anterior teeth are particularly important for grasping and tearing in a nature felid 288 diet, analyses were performed comparing just the anterior teeth of captive and wild felids. As 289 with the other analyses, the findings were significant in regards to the captive specimens having 290 higher calculus and periodontal scores (Table 6). The anterior teeth likely experience a high 


\section{Kapoor et al. Oral Health Correlates of Captivity}

291 amount of abrasion during hunting and also during consumption of tough tissue in whole

292 carcasses (Hartstone-Rose, 2008). In the captive specimens, these acts are completely removed

293 and the negative effect is clearly evident on the anterior teeth. While captivity has a negative

294 effect on all sections of the oral cavity, it seems likely that anterior oral health in particular is

295 worsened by the lack of tearing and grasping in a captive diet.

296 These results are likely due to the substantially different mechanical properties of the wild

297 and captive diets. The high rates and levels of calculus and periodontal disease in captive animals

298 are likely due to the lack of abrasion that accompanies chewing on bones or connective tissues.

299 Further, soft diets tend to produce more bacterial plaque than firm diets resulting in an increased

300 calculus accumulation and eventually gingivitis or periodontitis (Pearson et al., 2005).

301 Another potential cause of the higher incidences of dental calculus and periodontal disease

302 could be stress from living in captivity. There is an extensive literature documenting that animals

303 living in captivity experience significantly higher levels of stress indicators than their wild

304 counterparts (Bayazit, 2009; Burgener et al., 2008; Morgan and Tromborg, 2007; Terio et al.,

305 2004), and even further research that concludes that stress or captivity can affect an animal's

306 health (Baker et al., 1998; Constable et al., 1998; Kilbourn et al., 2003; Terio, 2009; Whitehouse-

307 Tedd et al., 2015). Yet, among all this research we were unable to find any that directly correlated

308 captivity stress to oral health. There are however, studies that support stress as a causative agent

309 for a deficiency in dental health in humans (Deinzer et al., 2005; Marcenes and Sheiham, 1992;

310 Muhvic-Urek et al., 2007). Therefore, it is possible that the increase in calculus and periodontal

311 disease in the captive specimens was a result of stress. 
Kapoor et al.

There are, of course, a variety of other factors that could potentially contribute to the

313 change in oral health noted in captivity. For example, in black-footed ferrets (Mustela nigripes)

314 concerns arose over the health effects of a lack of genetic diversity in the captive population

315 resulting from near extinction of the species (Wisely et al., 2002a). A similar study to the one

316 reported here found that oral health was worse among these captive black-footed ferrets

317 (Antonelli et al., 2015). But, a separate study that found decreased cranial sizes in captive black-

318 footed ferrets concluded that inbreeding was not statistically the cause of cranial morphological

319 differences (Wisely et al., 2002b), although this may not be the case in felids. There are an array

320 of factors that should be considered when looking at the effects of captivity on oral health, and

321 multiple factors could contribute simultaneously. However, the findings of this study are in

322 agreement with other literature (Fagan, 1980; Haberstroh et al., 1984; Logan, 2006; Watson,

323 1994) suggesting the mechanical properties of diet are a leading cause, if not the single greatest

324 cause for the deterioration of oral health in captive felids.

Effect of Oral Health on Overall Health

The possibility of damage to other organs and tissues as a consequence of periodontal

328 disease has been a topic of study for some time (Audet et al., 2002; McGrew, 1979). Although

329 changes in nutritional aspects of the diet have arguably improved the health of domestic cats and

330 dogs, periodontal disease remains a serious problem (Walton and Joly, 2003). Scientists and

331 dental professionals have long suspected associations between oral health and systemic health. In

332 humans, an association has been found between periodontal disease and diabetes, cardiovascular

333 disease, and pregnancy complications (Logan, 2006). Although the nature of the relationship is

334 not fully understood, studies thus far clearly point to a connection between periodontal health and

335 systemic health (Campbell, 2006).

336 This study demonstrates a direct relationship between captivity status and oral health. Our

337 comparison of periodontal disease and calculus occurrence to degree of cranial deformation - 
which is also correlated with captivity status (Hartstone-Rose et al., 2014) - in the same valuable information to keepers of captive animals regarding possible side effects that can arise

342 from oral health defects in captivity.

\section{Health of Anterior versus Posterior Dentition}

345 Because different regions of the dentition serve different functions during food

346 acquisition and processing, one aim of this study was to determine if variation in oral health

347 exists between the posterior and anterior dentition of captive and wild felids. Our results show a

348 highly correlated relationship between the oral health scores of the posterior and anterior teeth

349 (Table 8) - animals with poor anterior oral health also have poor posterior oral health. However,

350 calculus buildup is greater in the posterior teeth when compared to the anterior teeth, but the

351 opposite trend was seen for periodontal disease; higher prevalence of periodontal disease was

352 noted in the anterior teeth when compared to the posterior teeth (Table 8). Because carnivores

353 primarily use their anterior teeth for grasping and tearing, this action may damage the gingiva,

354 leading to inflammation and if not treated could lead to periodontal disease. This could explain

355 why periodontal disease is more prevalent on the anterior teeth. Higher prevalence of calculus

356 accumulation on the posterior teeth could be explained by the previous finding that plaque tends

357 to accumulate at the openings of parotid salivary ducts which are located near the cheek teeth

358 (Vosburgh et al., 1982).

\section{Effects of Age, Sex and Species}

361 One of the limitations of this study was that while the prevalence and severity of oral

362 health issues are age-related (Albuquerque et al., 2012; Gawor et al., 2006; Patterson et al., 2003;

363 Watson, 1994), the exact ages of the specimens in our study were unknown. However, a study by 
Kapoor et al. $\quad$ Oral Health Correlates of Captivity

364 Gawor and colleagues (2006) on the influence of diet on oral health of cats and dogs showed that

365 even after they adjusted for age, the mean oral health index was significantly higher in cats and

366 dogs fed soft food compared with those fed dry or mixed food. These results clearly indicate that

367 feeding a dry food diet has a positive influence on oral health (Walton and Joly, 2003).

368 Furthermore, we have found juvenile captive specimens with advanced oral health diseases (Fig.

3693 ), demonstrating that although age might be a confounding factor in this study, it is not

370 necessarily the main correlate of dental disease. Regardless, only adult specimens were included

371 in our study.

372

373 Insert Figure 3 Here

374

Results of one-way ANOVA tests showed no significant influence of sex and

376 species on oral health. The one statistical exception - tigers having significantly higher

377 maximum periodontal disease scores - is likely a statistical sample artifact since there is

378 no obvious biological reason that this should be true. This difference in maximum

379 periodontal disease score probably arose because of the large number of captive tigers

380 compared to wild tigers driving the mean scores of the tigers higher than those of the

381 lions which had a more equal distribution between captive and wild specimens. This is

382 supported by our further analysis comparing wild tigers to wild lions and captive tigers to

383 captive lions. When captivity status is adjusted for, there were no significant differences

384 between the two species. This not only implies that there is no notable difference between

385 the species, but also further supports our previous hypothesis that captivity is the

386 causative agent for oral health problems in both lions and tigers.

387

\section{$388 \quad$ Future Directions}


Although ground meat, soft diets are the standard among North American

390 captivity centers, this diet is not the predominant one fed in all global regions.

391 Specifically, European captivity centers tend to favor whole carcass feeding both for the

392 nutritional value of a natural diet, and also for the enrichment of the animals (Pearson et

393 al., 2005). An expansion of this study to examine oral health and cranial morphology

394 among specimens fed a whole carcass diet would provide strong comparative data

395 between the differences in effect on oral health among the two diet types. Additionally, a

396 comparison of specimens fed similar diets, but in different global areas (i.e. North

397 America and Europe), could help clarify whether the previously seen oral health issues

398 are truly diet induced or rather a factor of other zoo keeping characteristics prevalent in

399 North America.

400 The exact diet of the captive specimens could not be ascertained prior to the

401 study. Given that all the specimens are from North American captivity centers, however,

402 it is reasonable to surmise that the diet was indeed a ground meat diet. While this cannot

403 be stated with absolute certainty, a similar study of black-footed ferrets found the same

404 result - worse oral health in captive animals (Antonelli et al., 2015). Further still,

405 experimental data exist that point directly to diet composition as the cause of oral health

406 problems in captive timber wolves and even captive Amur tigers (Haberstroh et al., 1984;

407 Vosburgh et al., 1982). Further studies could be performed with the use of live specimens

408 to generate a more specific understanding of the exact effects of diet composition and

409 oral health in these large felid species.

410 Another direction in which to continue this work will be to examine the effects of

411 stress on oral health among captive animals. As previously mentioned, evidence supports

412 that captivity has negative health effects on animals, but there is no literature directed

413 specifically at oral health. An examination of live animals' dentition, as well as markers 
Kapoor et al. $\quad$ Oral Health Correlates of Captivity

414 of stress such as behavioral abnormalities or levels of cortisol, could provide information

415 on the relationship between the stresses of captivity and oral health.

416

417 Broader Impacts

418 The results of this study strongly suggest that the mechanical properties of diet are

419 important to oral health in felids. The wild felid diet, which allows more abrasive material (such

420 as bone, tendon, and skin) to contact the teeth, is correlated with a lower incidence of oral

421 disease. These data support recommendations for improvement in North American captive

422 carnivore diets from a mechanical perspective (Hartstone-Rose et al., 2014). Management of

423 dental disorders provides animals with a higher quality of life, extends their life span and

424 improves breeding capabilities (Van Foreest, 1993).

425 This study also demonstrates a link between oral health and cranial deformation. Further

426 understanding of the possible relationship between periodontal health and other systemic

427 conditions will allow zoo and veterinary professionals to provide the best overall health care for

428 captive carnivores.

\section{ACKNOWLEDGEMENTS}

430 Thanks to Drs. Edie Goldsmith, Chandrashekhar Patel, and two anonymous reviewers for 431 feedback on this manuscript and to Amanda Heckler, Kristen MacNeill, Brett Donadeo, and

432 Lucas Hartstone-Rose, for assisting in data collection. We also would like to thank the American

433 Museum of Natural History, the Smithsonian Institution's National Museum of Natural History, 434 and their staff (especially Eileen Westwig, Darrin Lunde, and John Ososky) for access to their 435 collections.

436 
Kapoor et al.

$\underline{\text { References }}$

Albuquerque, C., Morinha, F., Requicha, J., Martins, T., Dias, I., Guedes-Pinto, H., Bastos, E., Viegas, C., 2012. Canine periodontitis: the dog as an important model for periodontal studies. The Veterinary Journal 191, 299-305.

Antonelli, T., Leischner, C., Ososky, J., Hartstone-Rose, A., 2015. The effect of captivity on the oral health of the critically endangered black-footed ferret (Mustela nigripes). Canadian Journal of Zoology 94, 15-22.

Audet, A.M., Robbins, C.B., Larivière, S., 2002. Alopex lagopus. Mammalian Species, 110.

Baker, M.L., Gemmell, E., Gemmell, R.T., 1998. Physiological changes in brushtail possums, Trichosurus vulpecula, transferred from the wild to captivity. Journal of Experimental Zoology 280, 203-212.

Bayazit, V., 2009. Evaluation of cortisol and stress in captive animals. Aust J Basic Appl Sci 3, 1022-1031.

Bechert, U., Mortenson, J., Dierenfeld, E.S., Cheeke, P., Keller, M., Holick, M., Chen, T.C., Rogers, Q., 2002. Diet composition and blood values of captive cheetahs (Acinonyx jubatus) fed either supplemented meat or commercial food preparations. Journal of Zoo and Wildlife Medicine 33, 16-28.

Bowers, K., 2014. Carcass controversy a fact of life for some, UT San Diego. UT San Diego, Web.

Brown, M., Park, J., 1968. Control of dental calculus in experimental beagles. Laboratory animal care 18, 527.

Burgener, N., Gusset, M., Schmid, H., 2008. Frustrated appetitive foraging behavior, stereotypic pacing, and fecal glucocorticoid levels in snow leopards (Uncia uncia) in the Zurich Zoo. Journal of Applied Animal Welfare Science 11, 74-83.

Busscher, H.J., White, D.J., Kamminga-Rasker, H.J., van der Mei, H.C., 2004. A surface physicochemical rationale for calculus formation in the oral cavity. Journal of crystal growth 261, 87-92.

Campbell, E., 2006. It's more than the mouth: the effects of periodontal disease on systemic health. Dental assistant (Chicago, Ill.: 1994) 76, 26-28, 30-21.

Constable, P., Hinchcliff, K., Demma, N., Callahan, M., Dale, B., Fox, K., Adams, L., Wack, R., Kramer, L., 1998. Serum biochemistry of captive and free-ranging gray wolves (Canis lupus). Journal of Zoo and Wildlife Medicine, 435-440.

Deinzer, R., Granrath, N., Spahl, M., Linz, S., Waschul, B., Herforth, A., 2005. Stress, oral health behaviour and clinical outcome. British journal of health psychology 10, 269-283.

Dog, B., 2005. Published 20 December 2005 by the American Society of Mammalogists. Fagan, D.A., 1980. Diet consistency and periodontal disease in exotic carnivores, Proc. Am. Assoc. Zoo Vet, pp. 34-37.

Gawor, J.P., Reiter, A.M., Jodkowska, K., Kurski, G., Wojtacki, M.P., Kurek, A., 2006. Influence of diet on oral health in cats and dogs. J Nutr 136, 2021s-2023s.

Haberstroh, L.I., Ullrey, D.E., Sikarski, J.G., Richter, N.A., Colmery, B.H., Myers, T.D., 1984. Diet and Oral Health in Captive Amur Tigers (Panthera tigris altaica). Journal of Zoo Animal Medicine 15, 142-146. 
Kapoor et al.

482

483

484

485

486

487

488

489

490

491

492

493

494

495

496

497

498

499

500

501

502

503

504

505

506

507

508

509

510

511

512

513

514

515

516

517

518

519

520

521

522

523

524

525

526

Hartstone-Rose, A., 2008. Evaluating the hominin scavenging niche through analysis of the carcass-processing abilities of the carnivore guild, Biological Anthropology and Anatomy. Duke University, Durham, p. 190.

Hartstone-Rose, A., Perry, J., Morrow, C.J., 2012. Bite force estimation and the fiber architecture of felid masticatory muscles. The Anatomical Record 295, 13361351.

Hartstone-Rose, A., Selvey, H., Villari, J.R., Atwell, M., Schmidt, T., 2014. The ThreeDimensional Morphological Effects of Captivity. Plos One 9.

Kerr, K., Beloshapka, A., Morris, C., Parsons, C., Burke, S., Utterback, P., Swanson, K., 2013. Evaluation of four raw meat diets using domestic cats, captive exotic felids, and cecectomized roosters. Journal of animal science 91, 225-237.

Kilbourn, A.M., Karesh, W.B., Wolfe, N.D., Bosi, E.J., Cook, R.A., Andau, M., 2003. Health evaluation of free-ranging and semi-captive orangutans (Pongo pygmaeus pygmaeus) in Sabah, Malaysia. Journal of Wildlife Diseases 39, 73-83.

Lindburg, D.G., 1988. Improving the feeding of captive felines through application of field data. Zoo Biology 7, 211-218.

Logan, E.I., 2006. Dietary influences on periodontal health in dogs and cats. Veterinary Clinics: Small Animal Practice 36, 1385-1401.

Marcenes, W.S., Sheiham, A., 1992. The relationship between work stress and oral health status. Social Science \& Medicine 35, 1511-1520.

McGrew, J.C., 1979. Vulpes macrotis. Mammalian Species Archive 123, 1-6.

McPhee, M.E., 2002. Intact carcasses as enrichment for large felids: Effects on on- and off-exhibit behaviors. Zoo Biology 21, 37-47.

Morgan, K.N., Tromborg, C.T., 2007. Sources of stress in captivity. Applied Animal Behaviour Science 102, 262-302.

Muhvic - Urek, M., Uhač, I., Vuksic - Mihaljevic, Ž., Leović, D., Blečić, N., Kovač, Z., 2007. Oral health status in war veterans with post - traumatic stress disorder. Journal of Oral Rehabilitation 34, 1-8.

Niemiec, B.A., 2008a. Oral pathology. Topics in companion animal medicine 23, 59-71.

Niemiec, B.A., 2008b. Periodontal disease. Topics in companion animal medicine 23, 7280.

Patterson, B.D., Neiburger, E.J., Kasiki, S.M., 2003. Tooth breakage and dental disease as causes of carnivore-human conflicts. Journal of Mammalogy 84, 190-196.

Pearson, R., Knight, K., Melfi, V., Nicklin, A., 2005. Does the provision of carcasses compromise the health of zoo-housed carnivores? Preliminary report, Proceedings of the 7th Annual Symposium on Zoo Research, Twycross Zoo, Warwickshire, UK, 7-8th July 2005. British and Irish Association of Zoos and Aquariums, pp. 194-199.

Pihlstrom, B.L., Michalowicz, B.S., Johnson, N.W., 2005. Periodontal diseases. The Lancet 366, 1809-1820.

Roberts, M.S., Gittleman, J.L., 1984. Ailurus fulgens. Mammalian Species Archive 222, $1-8$.

Samuelson, A.C., Cutter, G., 1991. Dog biscuits: an aid in canine tartar control. The Journal of Nutrition 121, S162-S162.

Schaller, G., 1972. The Serengeti Lion. University of Chicago Press, Chicago. 
Kapoor et al.

Schwarts, N.D., 2014. Anger Erupts After Danish Zoo Kills a "Surplus" Giraffe, New York Times. New York Times, New York Times, p. A6.

Skibiel, A.L., Trevino, H.S., Naugher, K., 2007. Comparison of several types of enrichment for captive felids. Zoo Biology 26, 371-381.

Sunquist, M., Sunquist, F., 2002. Wild Cats of the World University Of Chicago Press, Chicago.

Terio, K.A., 2009. Diseases of captive and free-ranging non-domestic felids. Fundacion Biodiversidad.

Terio, K.A., Marker, L., Munson, L., 2004. Evidence for chronic stress in captive but not free-ranging cheetahs (Acinonyx jubatus) based on adrenal morphology and function. Journal of Wildlife Diseases 40, 259-266.

Van Foreest, A., 1993. Veterinary dentistry in zoo and wild animals. Zoo and wild animal medicine, current therapy 3, 263-268.

Van Valkenburgh, B., 1989. Carnivore dental adaptations and diet: a study of trophic diverstiy within guilds, in: Gittleman, J.L. (Ed.), Carnivore behavior, ecology, and evolution. Cornell UP, Ithica, pp. 410-436.

Verstraete, F.J., 2003. Advances in diagnosis and treatment of small exotic mammal dental disease, Seminars in Avian and Exotic Pet Medicine. Elsevier, pp. 37-48.

Vester, B.M., Burke, S.L., Dikeman, C.L., Simmons, L.G., Swanson, K.S., 2008. Nutrient digestibility and fecal characteristics are different among captive exotic felids fed a beef-based raw diet. Zoo Biology 27, 126-136.

Vosburgh, K., Barbiers, R., Sikarskie, J., Ullrey, D., 1982. A soft versus hard diet and oral health in captive timber wolves (Canis lupus). The Journal of Zoo Animal Medicine, 104-107.

Walton, L.R., Joly, D.O., 2003. Canis mesomelas. Mammalian Species, 1-9.

Watson, A.D.J., 1994. Diet and periodontal disease in dogs and cats. Australian Veterinary Journal 71, 313-318.

Whitehouse-Tedd, K.M., Lefebvre, S.L., Janssens, G.P., 2015. Dietary Factors Associated with Faecal Consistency and Other Indicators of Gastrointestinal Health in the Captive Cheetah (Acinonyx jubatus). Plos One 10, e0120903.

Wiggs, R.B., Bloom, B.C., 2003. Exotic placental carnivore dentistry. Veterinary Clinics of North America: Exotic Animal Practice 6, 571-599.

Wisely, S., Buskirk, S., Fleming, M., McDonald, D., Ostrander, E., 2002a. Genetic diversity and fitness in black-footed ferrets before and during a bottleneck. Journal of Heredity 93, 231-237.

Wisely, S., Ososky, J., Buskirk, S., 2002b. Morphological changes to black-footed ferrets (Mustela nigripes) resulting from captivity. Canadian Journal of Zoology 80, 1562-1568. 


\section{$567 \quad$ Figure Captions}

568 Figure 1. Analytical sections for the mandibular (A.) and maxillary (right) teeth.

569 Figure 2. Percent of specimens exhibiting some (score > 0) and moderate-to-extreme (score

$570>$ 1) of mean and maximum calculus and periodontal disease scores. Gray bars = wild

571 specimens; black bars = captive specimens.

572 Figure 3. Severe calculus (and moderate periodontal disease) on maxillary premolars of a

573 juvenille tiger (USNM 396272). Note the deciduous premolar being replaced by the permanent

574 premolar. As only adult specimens were included in the statistical analyses, this specimen is used

575 here only for illustrative purposes.

576 
Kapoor et al. Oral Health Correlates of Captivity

577 Table 1. Number of specimens in this sample broken down by species, captivity status, and

$578 \operatorname{sex}(n=63)$

\begin{tabular}{cccccc}
\hline $\begin{array}{c}\text { Common } \\
\text { Name }\end{array}$ & Species & Captive (Zoo) & Wild & Male & Female \\
\hline Lion & Panthera & 15 & 19 & 17 & 17 \\
& leo & & & & \\
Tiger & Panthera & 20 & 9 & 14 & 15 \\
& tigris & & & & \\
& TOTAL & 35 & 28 & 31 & 32 \\
\hline
\end{tabular}

579

580 
Kapoor et al.

Oral Health Correlates of Captivity

581

Table 2. Calculus Index

Calculus

Scores

Observations

\begin{tabular}{ll}
\hline 0 & No evidence of calculus \\
1 & $1-10 \%$ of tooth crown is covered by calculus \\
2 & $10-25 \%$ of tooth crown is covered by calculus \\
3 & $25-50 \%$ of tooth crown is covered by calculus \\
4 & $50-75 \%$ of tooth crown is covered by calculus and/or minor caries \\
5 & $75-100 \%$ of tooth crown is covered by calculus OR thickened calculus and/or \\
& major caries
\end{tabular}

582

583 
Kapoor et al.

Oral Health Correlates of Captivity

584 Table 3. Periodontal Disease Index

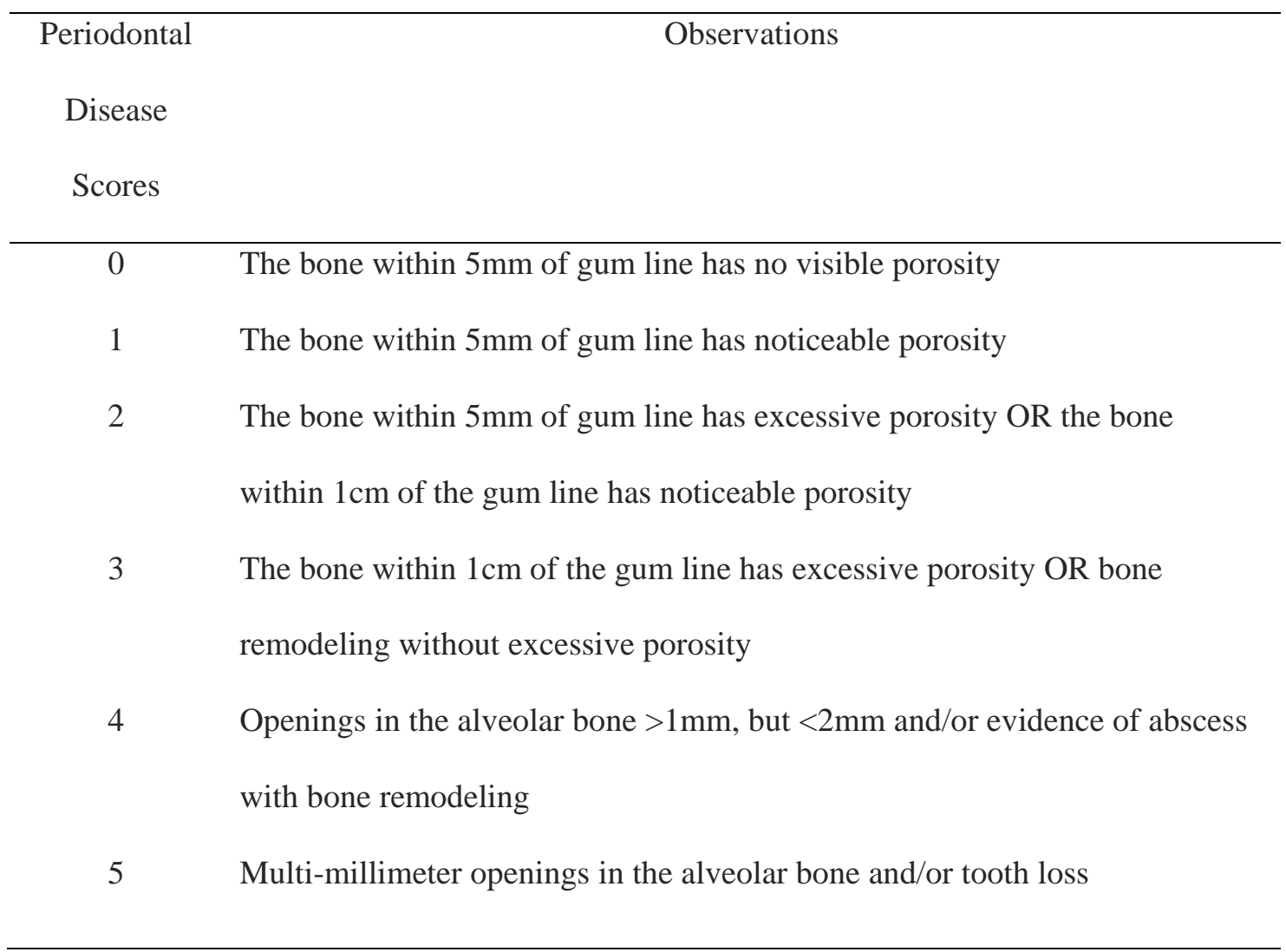

585

586 
Kapoor et al. Oral Health Correlates of Captivity

587 Table 4. Presence and absence of calculus and periodontal disease in wild and

588 captive felids.

\begin{tabular}{ccccc}
\hline Variables & Wild \% & Captive \% & Wild \% Score & Captive \% Score \\
& Score $>0$ & Score $>0$ & $>1$ & $>1$ \\
\hline Mean Calculus Score & $18 \%$ & $97 \%$ & $4 \%$ & $46 \%$ \\
Maximum Calculus Score & $18 \%$ & $97 \%$ & $4 \%$ & $34 \%$ \\
Mean Periodontal Score & $64 \%$ & $97 \%$ & $11 \%$ & $88 \%$ \\
Maximum Periodontal & $64 \%$ & $97 \%$ & $25 \%$ & $83 \%$ \\
Score & & & &
\end{tabular}

589

590 
Kapoor et al.

Oral Health Correlates of Captivity

591 Table 5. One-way ANOVA Analysis of Oral Health and Captivity Status.

\begin{tabular}{llll}
\hline Variable & Mean ${ }^{\mathrm{a}}(\mathrm{SD})$ & Mean $^{\mathrm{a}}(\mathrm{SD})$ & p-Value \\
& Zoo & Wild & \\
\hline Mean ${ }^{\mathrm{b}}$ Periodontal & $1.62(0.75)$ & $0.39(0.53)$ & $<.0001$ \\
Disease & & & \\
& & & \\
Maximum $^{\mathrm{c}}$ & $3.08(1.40)$ & $1.00(1.09)$ & $<.0001$ \\
Periodontal Disease & & & \\
& & & \\
Mean $^{\mathrm{b}}$ Calculus $^{1.05(0.66)}$ & $0.08(0.21)$ & $<.0001$ \\
& & & \\
Maximum $^{\mathrm{c}}$ Calculus & $1.77(1.33)$ & $0.21(0.49)$ & $<.0001$
\end{tabular}

$592 \quad{ }^{a}$ Average score across all specimens.

$593{ }^{\mathrm{b}}$ Average score of all six dental sections in each specimen.

$594{ }^{c}$ Highest score of the six dental sections per specimen.

595 
Kapoor et al.

Oral Health Correlates of Captivity

596 Table 6. One-way ANOVA Analysis of Anterior Oral Health and Captivity Status.

\begin{tabular}{llll}
\hline Variable & Mean $^{\mathrm{a}}(\mathrm{SD})$ & Mean $^{\mathrm{a}}(\mathrm{SD})$ & p-Value \\
& Zoo & Wild & \\
& & & \\
\hline Upper Anterior & $2.60(0.18)$ & $0.36(0.21)$ & $<.0001$
\end{tabular}

\begin{tabular}{llll}
\hline Upper Anterior & $2.60(0.18)$ & $0.36(0.21)$ & $<.0001$
\end{tabular}

Periodontal Disease

Lower Anterior $\quad 2.11(0.15) \quad 0.71(0.17) \quad<.0001$

Periodontal Disease

$\begin{array}{lll}\text { Upper Anterior } \quad 0.71(0.11) & 0.11(0.13) \quad .0008\end{array}$

Calculus

Lower Anterior $\quad 0.46(0.07) \quad 0.04(0.08) \quad<.0001$

Calculus

$597 \quad{ }^{a}$ Average score across all specimens. 
Kapoor et al.

Oral Health Correlates of Captivity

598 Table 7. Descriptive statistics for RMA regressions of dental scores against the captivity

599 coefficient.

\begin{tabular}{lcccccc}
\hline Dependent variable & Slope & y-intercept & $\mathrm{r}^{2}$ & Lower CL & Upper CL & p-Value \\
\hline Mean Periodontal & 34.04 & 1.09 & 0.42 & 18.22 & 63.61 & $>0.0006$ \\
Maximum & 61.84 & 2.19 & 0.48 & 37.52 & 101.94 & $>0.0001$ \\
Periodontal & & & & & & \\
Mean Calculus & 26.65 & 0.63 & 0.41 & 13.84 & 51.33 & $>0.0009$ \\
Maximum Calculus & 49.03 & 1.11 & 0.33 & 19.59 & 122.71 & $>0.0076$ \\
& & & & & & \\
\hline
\end{tabular}

600

601 
Kapoor et al. Oral Health Correlates of Captivity

602 Table 8. Descriptive statistics for RMA regressions of the anterior (independent) and

603 posterior (dependent) regions by dental score.

\begin{tabular}{ccccccc}
\hline Variable & Slope & y-intercept & $\mathrm{r}^{2}$ & Lower CL & Upper CL & p-Value \\
\hline Periodontal Disease & 0.70 & -0.24 & 0.38 & 0.50 & 0.98 & $<.0001$ \\
Scores & & & & & & \\
& & & & & & \\
Calculus Scores & 1.64 & 0.14 & 0.35 & 1.14 & 2.366 & $<.0001$ \\
\hline
\end{tabular}

604

605 

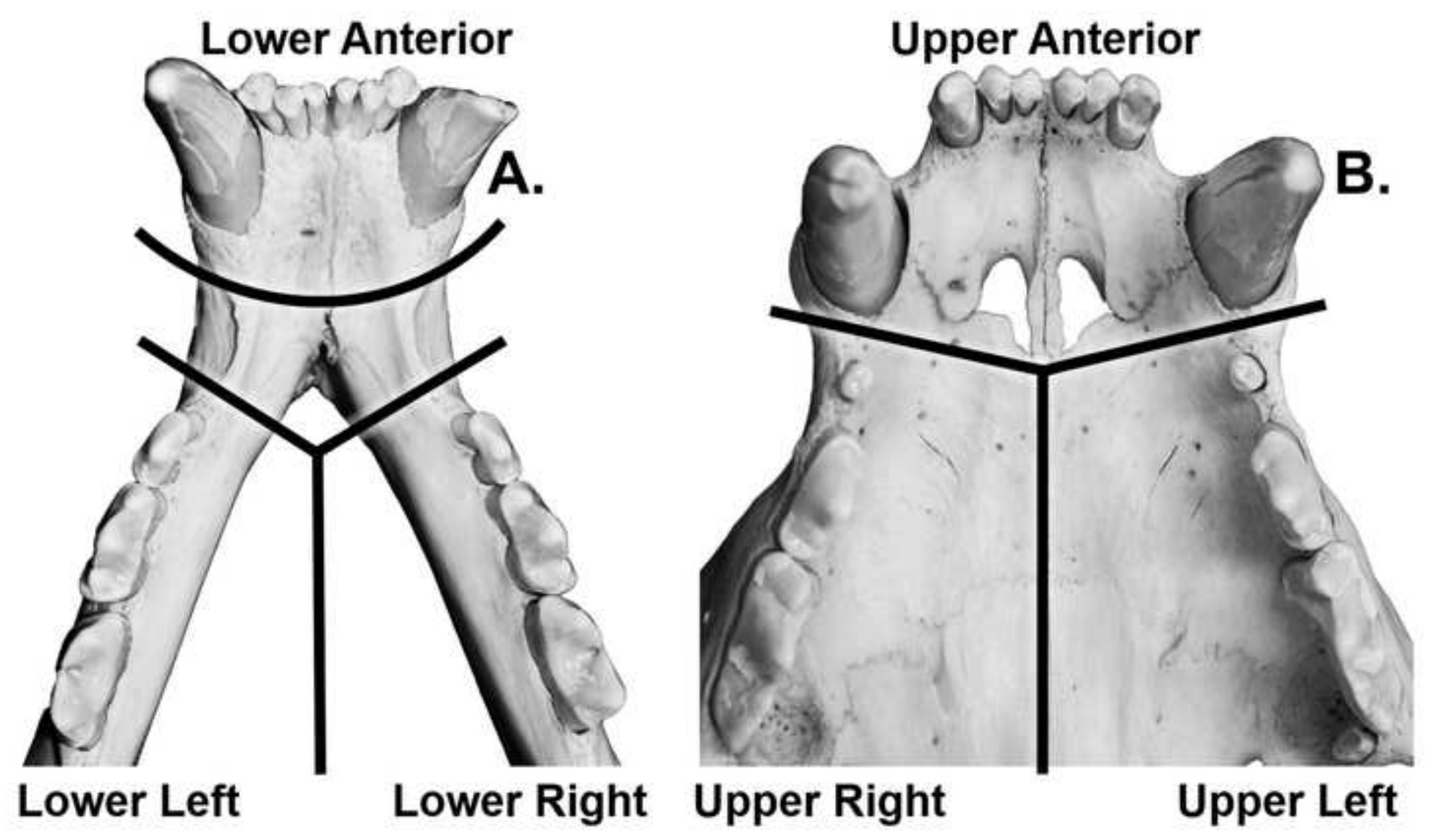
$100 \%$

$90 \%$

$80 \%$

$70 \%$

$60 \%$

$50 \%$

$40 \%$

$30 \%$

$20 \%$

$10 \%$

$0 \%$

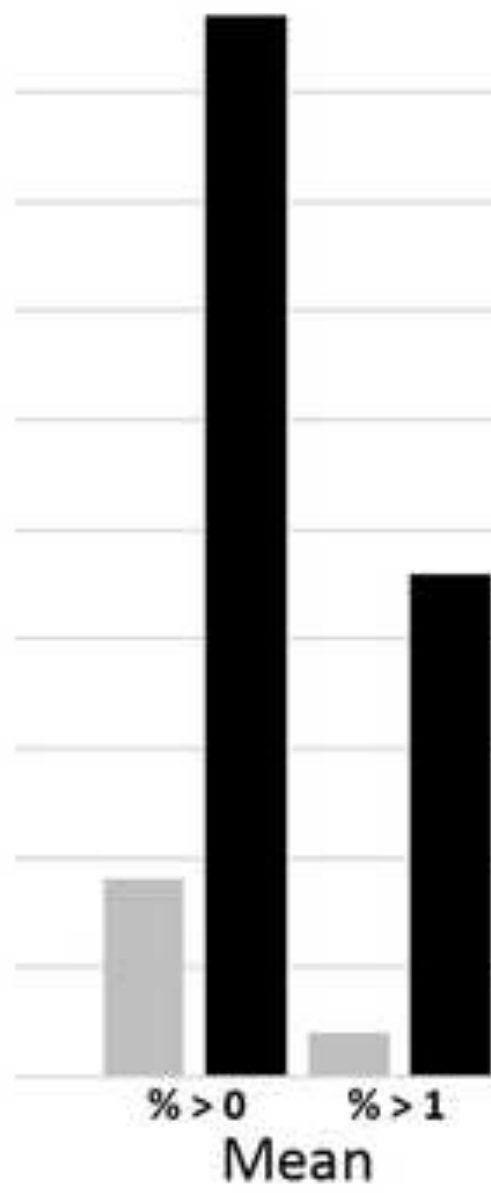

Calculus Score

$\%>0$

Maximum

Calculus Score

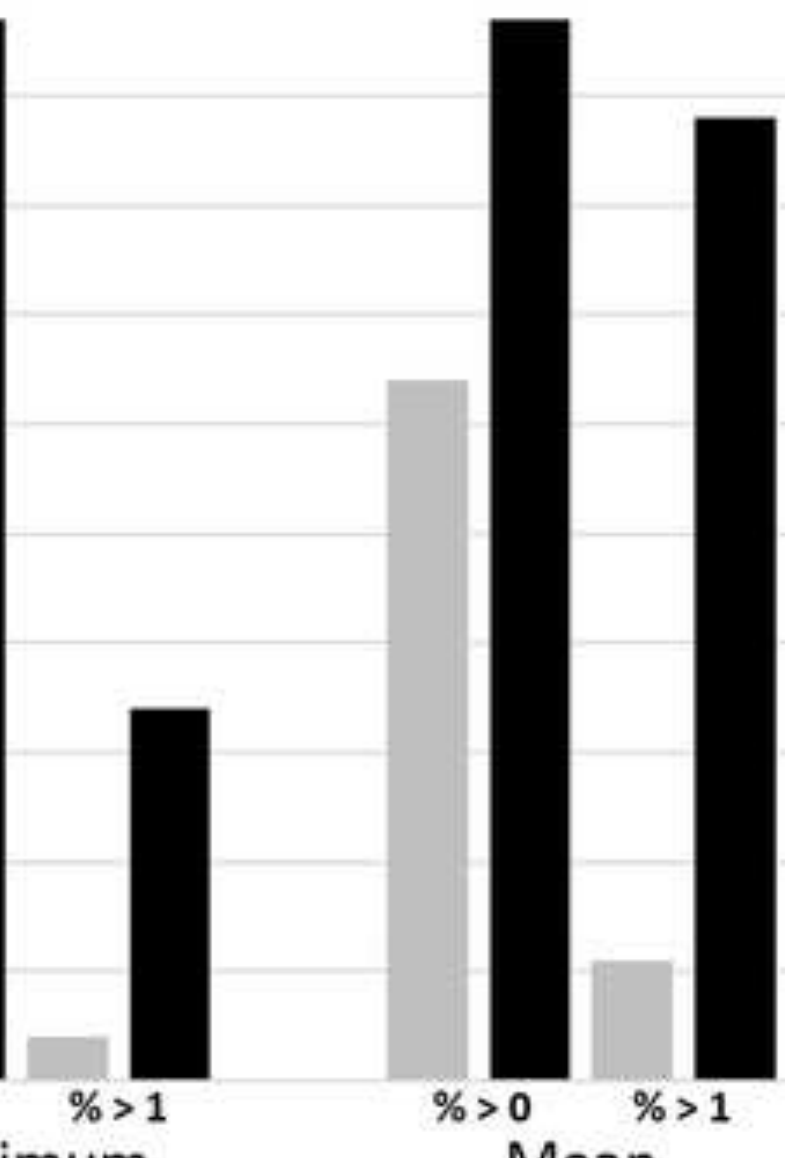

Mean

$\%>0 \quad \%>1$

Maximum

Periodontal Score Periodontal Score 


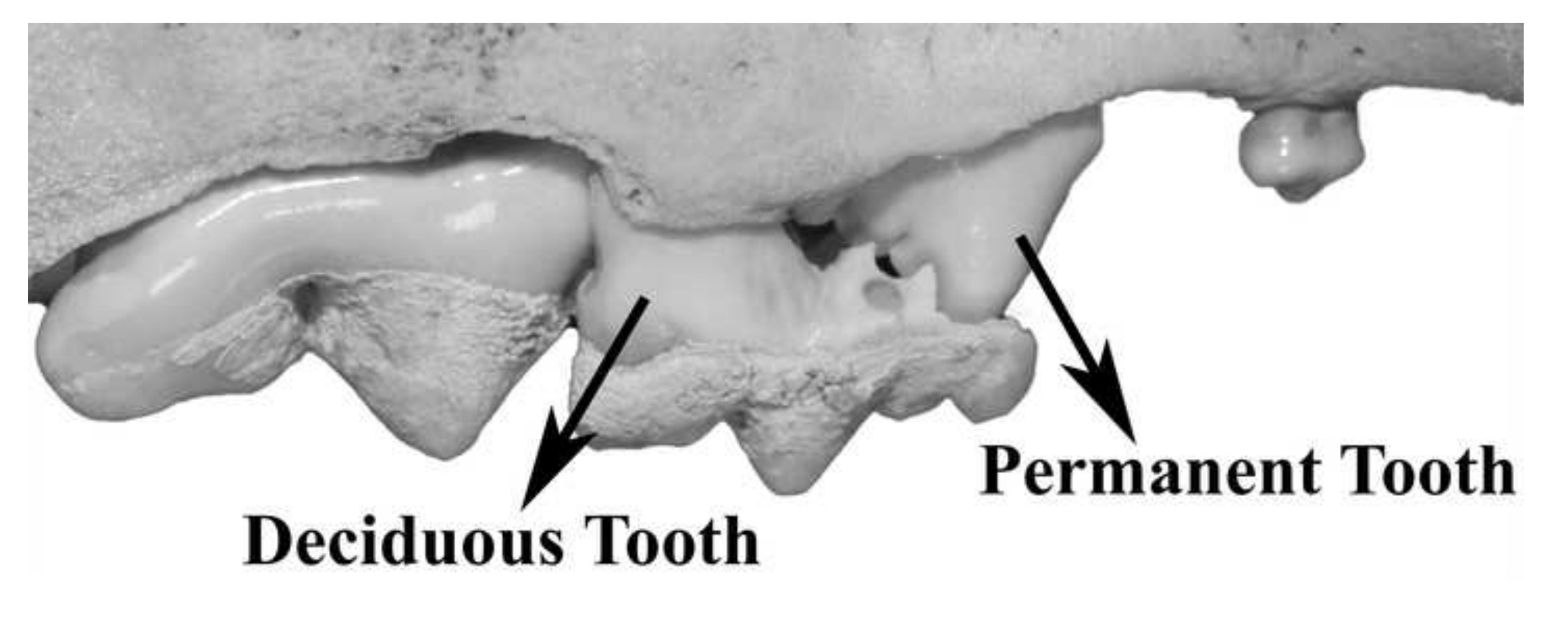

Deciduous Tooth
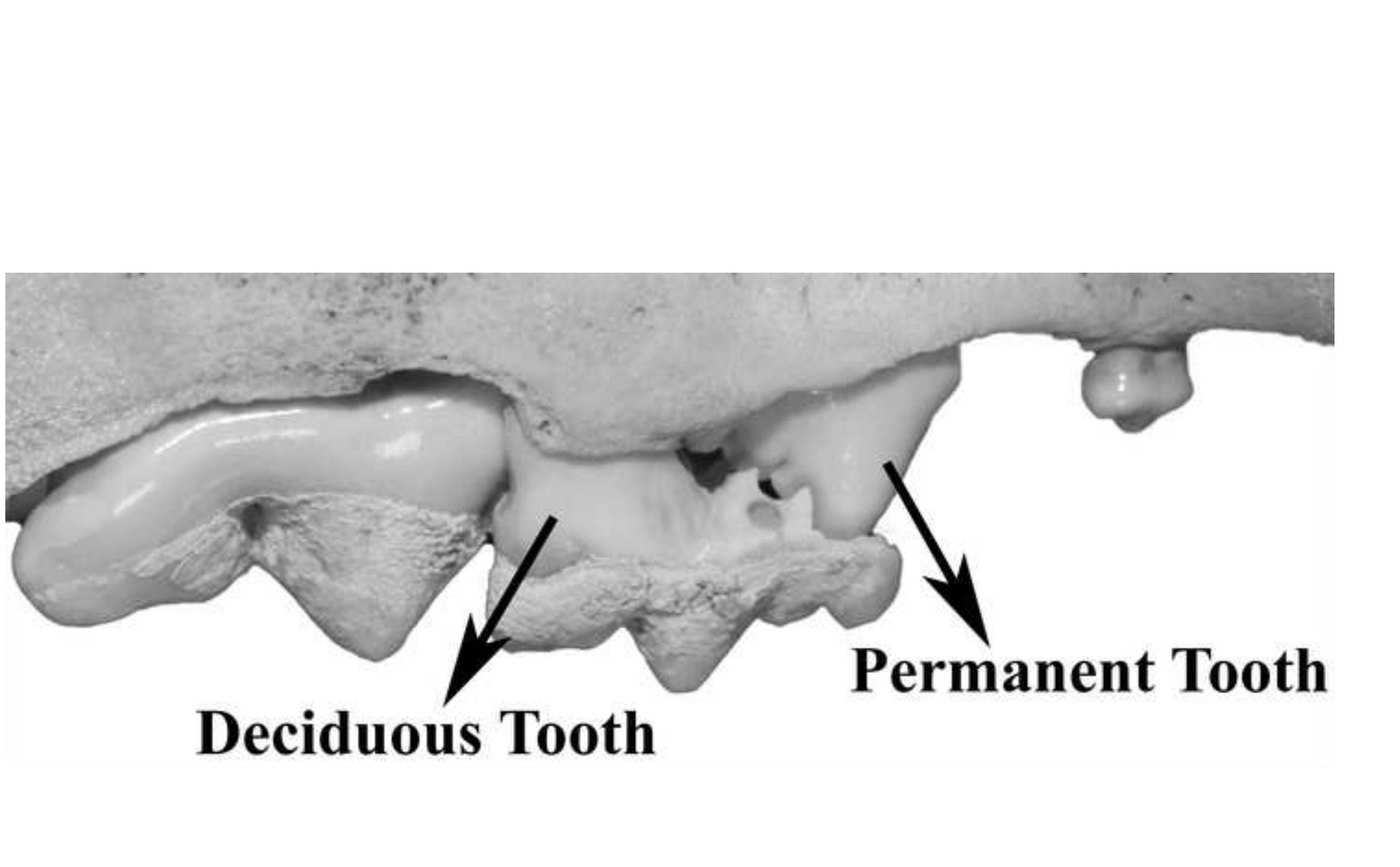

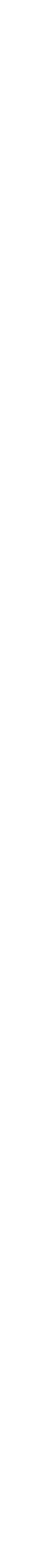
.
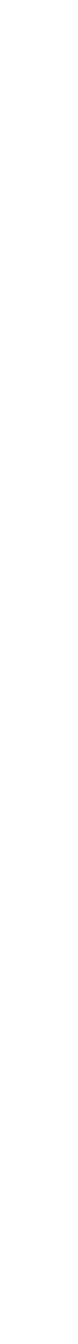